\title{
The Value of European Exchange Programs for Early Career Family Doctors
}

Bernadeta Bridgwood*, Academic Clinical Fellow in General Practice, b.bridgwood@nhs.net Hannah Willoughby, General Practitioner

Madeleine Attridge, General Practitioner

Eugene Tang, Academic General Practitioner Registrar, e.y.h.tang@newcastle.ac.uk

\section{*Corresponding author}

Dr Bernadeta Bridgwood

Centre for Medicine

University of Leicester

15 Lancaster Rd,

Leicester

LE1 7HA, UK

b.bridgwood@nhs.net

Dr Eugene Tang

Institute of Health and Society

Newcastle University

Level 2, Biomedical Research Building

Campus for Ageing and Vitality

Newcastle upon Tyne

NE4 5PL, UK

e.y.h.tang@newcastle.ac.uk 
Dr Hannah Willoughby,

Morlais Medical Practice,

Berry Square,

Dowlais,

Merthyr Tydfil

CF48 3AL

Hannahwilloughby2002@yahoo.co.uk

Dr Madeleine Attridge, Cwm Taf Primary Care support Unit Ynysmeurig House Navigation Park Abercynon CF45 4SN

Madz_g@hotmail.com 


\title{
The Value of Exchange Programs for Early Career Family Doctors
}

\begin{abstract}
:
The Hippokrates Exchange programme (HEP) is designed to give early career General Practitioners (Associates in Training (AITs)/First5s) first-hand experience into concepts of a primary healthcare system outside of their host country. It is hoped that this will improve GP knowledge and skills, inspire professional development and promote a global approach to primary care. Although an increasing number are taking up this opportunity, their views and experiences of international exchanges are not well described. The objective was, therefore, to explore the experiences of AITs/First5s following their HEP exchanges.
\end{abstract}

This qualitative study analysed 16 post-exchange reports of UK participants visiting 10 European countries via the HEP, using a thematic approach. Participants were unanimously positive about their exchange experiences. The themes that emerged were 1) Comparison of primary care practice 2) Infrastructure of host primary care 3) Motivation and experience of exchanges and 4) Learning and reflection.

Participants displayed considerable benefits from these exchanges by broadening their experience in different primary care systems and identifying improvements to be incorporated into their UK practice. (Word count 170)

\section{Acknowledgements}

To the Junior International Committee of the RCGP and International Programme Board of the RCGP.

\section{Conflicts of interest}

None all authors 


\section{Funding}

Eugene Tang is supported by a\# NIHR Doctoral Research Fellowship (DRF-2015-08-006)

\section{Disclosure statement}

None to disclose

\section{Keywords/MESH terms}

General practice

Family practice

Exchange

Qualitative

Hippokrates

\section{Key messages}

1. Qualitative analysis of reports from exchange participants suggest a positive and rewarding international experience

2. Thematic analysis revealed that participants compare and evaluate primary care practice and infrastructure and identify potential areas for improvement within their own practice.

\section{Introduction}

International mobility has been adopted by many disciplines as an instrument to facilitate European integration $(1,2)$. The motivations of initiatives such as town-twinning projects and cross-European research are to align commonalities, promoting a supranational identity (3). 
Economically and educationally, the benefits of international experience are wide-reaching from learning a new language (4) to forging international research partnerships (5).

In the UK, overseas 'elective’ placements are common amongst medical undergraduates, but more recently international mobility has expanded into post-graduate medical education and general practice. This follows the recognition of an increasingly multicultural population with multiple health conditions and complex social needs (6). This presents training and service provision challenges for the modern healthcare system $(7,8)$. Providing the opportunity to directly experience how general practice is delivered in other European countries is a vital addition to training. Doctors are nurtured to better deliver medicine in a multicultural society and workplace $(6,7)$.

The Royal College of General Practitioners (RCGP) recognised the need for modernising GP training to reflect the culturally diverse healthcare environment. As such, the RCGP currently offer their trainees/young GPs the opportunity to participate in an international exchange via the HEP scheme, using support from European Funding (9). This takes the form of 2 week observership placement. One of the great strengths of this project is that learning is individualised; learning objectives are pre-agreed by the visitor and host. Each participant follows a formally agreed educational timetable and informally learns through social interaction with local doctors and trainees. Upon completion, each participant is required to write a report of their experience.

Although anticipated that doctors participating in exchanges gain knowledge that contributes to their wider-medical knowledge, it is unclear what value UK participants place on such exchange experiences and how it influences their clinical practice. The objective of this study 
was to explore the experiences of AiTs/First5 following international exchanges, using content analysis to investigate the following research questions:

1) What are the reported experiences of early career general practitioners when undertaking international exchange programs?

2) What do these reports show us about the expressed views of the respondents on the value of participating in international primary care focused exchange programs?

\section{Methods}

\section{Design and Setting}

Exchange participants (AiTs and First5s) were required to produce an exchange report after completing their 2-week exchange - a condition of accepting the exchange. Participants were given a standard set of questions (Appendix 1). Two participants also attended a conference as part of their exchange. Sixteen from a total of twenty-three participants who completed a report in the timeframe, provided informed consented to take part. It is unclear why remaining participants did not respond to the request for consent. Data was collected from exchanges between April 2010 and April 2013.

\section{Document review}

Sixteen unstructured participant reports were reviewed to deduce common learning themes. Reports were anonymised prior to data analysis. To improve inter-rate reliability, the data set was interrogated individually by two independent researchers (HW, BB). Ethical approval was given from Cardiff University School of Medicine Ethics committee.

\section{Data Analysis}


Here grounded theory was applied to facilitate the interpretation of the data set (10), wherEach report was subjected to eA-data--driven, $\mathrm{c}$ - - -ontent analysis-using an inductive approach was applied. The authors familiarised themselves with the data set by reading through the reports. The reports were then subjected to line-by-line coding independently and managed by NVIVO 11 . These codes were then divided into various main themes and subthemes-towe identifyied themes and subthemes from the reports._-Themes were subsequently grouped into broad categories following discussion between authors to facilitate interpretation of the data.

The program NVivo 11 was used to aid the data assessment.

\section{Results}

Table 1 summarises the characteristics of the sixteen participant reports, including the countries visited. All participants had undertaken an exchange based within primary care through HEP. the Hippokrates exchange programme.

From the data, four main themes emerged: Comparison of primary care practice, Infrastructure of primary care in the host country; Motivation and experience of the exchange and Learning and reflection.

\section{Theme 1 - Comparison of primary care practice}

All participants provided comparisons between home and host primary care systems, noting similarities and differences - particularly the healthcare structure, skills-set, funding allocation and utilisation of health insurance. Furthermore, specific cultural comparisons were made, often in relation to the style of general practice through to patient 
expectations/ideology. Participants evaluated whether positive differences in practice could potentially be adopted by the UK system. These prompted important reflections regarding their own practice and patients.

Report M (France) "We can also learn a lot from the rural French GPs who have the skills and ability to put less pressure on secondary care by providing services such as suturing and LARC insertion nearer to the patient's home."

Report G (Poland) “... in Poland, patients are able to access the following specialities without first seeing a GP; Gynaecology, (...) and Obstetrics. This was interesting as I had previously consulted a number of female Polish patients in the UK with standard primary care gynaecology problems, but they had been insistent on referral to a "specialist", and I am assuming that this is a reflection of how services operate in Poland."

Participants compared the GP training structure. Although the length was often compatible with the UK, it was often broader and provided more structured exposure to different specialities. Furthermore, assessment ranged from formal to informal.

Report H (Slovenia) “(Training includes)..... internal medicine... surgery... paediatrics... infectious diseases... obstetrics and gynaecology... psychiatry... orthopaedics, oncology, dermatology, neurology, ophthalmology and ENT."

Report P (France) "GP training in France is also three years, however there are no compulsory exams, like MRCGP.” 


\section{Theme 2 - Infrastructure of primary care in the host country}

Most participants discussed the infrastructure of the host GP practice. This usually included the number of GPs, trainees and multidisciplinary team members on the premises. List size varied from 600 to 17,000 patients, and in some cases whole families were assigned to one GP. Appointment length varied from 5-45 minutes and included direct contact, telephone and email contact. Participants visiting Spain, Portugal and Denmark commented on a decreased number of home visits, due to either patient expectation or nurses performing this role.

Report E (Portugal) "The core of the Portuguese general practice is the family list. At a family health centre (...) a single GP will have the entirety of a given household on his or her patient list, and each time the patient attends they will see this doctor."

Report D (Portugal) "Home visits are a rarity for doctors. However they are quite a routine for nurses.”

Report P (France) "The consultation length varied, with the average lasting 30 minutes."

Participants often discussed poor interaction with secondary care, including specialism within the community, difficulties referring into secondary care, and a lack of communication between primary and secondary care.

Report G (Poland) “What was immediately apparent was that the relationship with secondary care was poor... Despite these specialists being more community based, GPs didn't have close relationships with their secondary care colleagues."

Report H (Slovenia) "Communication between the hospital specialties and the GP following review of the patient by secondary care is by letter given to the patient and not directly to the GP.” 


\section{Theme 3 - Motivation and experience of the exchange}

Participants appeared to have similar motivations for undertaking an exchange. For most it was to learn about another primary healthcare system, including evaluating ideas and practice to incorporate into their own skills repertoire. Interestingly, none of the participants discussed an intention to experience the host country with the aim to work within it. Instead, most participants expressed a desire to bring the knowledge back to the UK system.

Report E (Portugal) "I am also fascinated by 'the consultation' and wanted to see whether there were many differences in the disease presentation, health beliefs and the relationship between doctor and patient in the two different countries."

Report K (Austria) "I return to the UK refreshed, and excited, having made some lifelong friends. I sincerely hope we stay in touch, and can support each other in our own personal goals and the goals for the healthcare we provide."

Participants were very emotive about the inclusion that hosts and sometimes patients, displayed, incorporating the participants into the wider multidisciplinary team.

Report F (Spain) "I was made very welcome at the health centre in Camps Blanc (...) Everyone was also very interested to discuss and compare and contrast our two health care systems and training programmes.”

Report N (Holland) "I was very warmly welcomed by my hosts and made to feel very much at ease in the practice. It was a very interesting and culturally enriching experience which I would recommend to all GP trainees." 


\section{Theme 4 - Learning and reflection}

All participants were asked to identify their learning objectives prior to the exchange and correlate their experience post-exchange. There was good evidence that the participants matured in their understanding of many aspects of primary care from individual patient consultations to overarching wider healthcare issues. Experiencing alternative forms of practice evoked discussion and identified potential areas of practice that could be translated into practice within the UK. Furthermore, participants demonstrated the development of translatable skills, such as improved understanding of cultural differences and communication.

Report J (Italy) "I can see the benefits of a structured healthcare system such as that in the UK and aim to learn more about the management side of general practice, particularly the specific role of the practice manager and work-related personnel issues."

Report M (France) “...this way of issuing scripts certainly encourages monitoring of patients on a regular basis.... I feel this is something I should certainly take note of when I return to Glasgow."

Report D(Portugal) "I believe there is much that we in the UK NHS can learn from sharing their experiences." $\mathrm{O}$ (Poland) "I have increased appreciation of cultural differences in those patients from other countries consulting with me..."

Participants discussed the exchange of information with hosts or peers and described a mutual enthusiasm to learn. Some participants extended this into research forums by attending secondary or primary care conferences. Indeed, there was optimism to disseminate their learning experience in peer reviewed publications.

Report N (Holland) "Similar to our UK VTS, the trainees have a session each week where 
they share interesting cases and work experiences. I was privileged to participate and able to tell them a little about general practice in the UK."

Report O (Poland) “As WONCA conference 2011 was held in Warsaw during the time of our exchange I travelled to Warsaw with 3 members of the general practice department of Jagiellonian University to the conference. I was lucky enough to have a poster accepted to present."

Report I (Netherlands) "This exchange was a great learning and reflective experience from both host and visitor perspectives. (...) I plan to publish further findings in one of the national primary care journals.”

\section{Discussion}

A global view is invaluable in primary care. There is an ever changing socioeconomic climate within medicine, particularly following mass mobility and migration across Europe $(5,11)$. This has identified the importance of promoting intercultural learning and preparing physicians treating diverse ethnic groups accessing UK primary care. GPs/trainee GPs need to be both clinically competent and culturally sensitive. GPs must be flexible in order to accommodate different social settings and deliver health care within multi-ethnic teams and healthcare settings. Indeed, there has been an increasing emphasis by policy makers to promote equality in healthcare access and outcomes among all population groups (12). Equally, reflecting on differences and similarities with our international neighbours provides important insight to evaluate and integrate new ideas to improve our own general practice. 
eommunity (13). There is an assumption that direct personal contact of differing cultural and national backgrounds can provide cooperation (13). Examining HEP,Hippokrates international exchanges, it was evident that international-mobility is an important component for education, insight, development and integration within our multi-cultural society. Globally aware GPs could provide patients in the UK with a more empathetic and personalised healthcare.

Qualitative analysis of these exchange reports-demonstrated-development of a participants with a greater cultural sensitivity and understanding, a key translatable skills in our multicultural society. fange of translatable skills both professional such as communication skills and empathy; and personal such as organisation and managerial. This satisfied the primary objectives of the exchange programme i.e. exchange and mobility and further participants gain knowledge and experience. This contributed to their training in line with key skills and core competencies outlined in the RCGP training curriculum.

Within UK general practice and a constantly restructuring NHS, young GPs need a broad perspective on health, management and leadership and commissioning services. Through international observational exchangesHEP, these young doctors had the opportunity to broaden their understanding and perspective of general practice issues. This study has provided an overview of the experiences of participants who undertook a two week HEP. The exchanges allowed participants to This allow compare and contrast practice between UK and international healthcare systems - importantly about potentially translatable areas of good practice. Experiential exchange learning within primary care has been demonstrated within other programs and other cultures. For example, Japanese participants gained knowledge in clinical, teaching and research skills from a UK exchange (14). Similarly, 
participants within an Irish-Australian exchange demonstrated improved procedural and educational skills (15).

\section{Strengths and Limitations}

This was the first qualitative evaluation-overview of the reports generated by early career GPs undertaking HEP. of international exchanges,-amongst early career GPs. We were able to identify the positive learning experience gained - both in terms of personal and professional attributes - and evidenced the value of international exchange. Observing delivery of general practice across Europe identified alternative and potentially improved practice methods to both the participant and the reader. With the integration of Global Health Fellowships into GP training [19], the value of these international experiences is being recognised. The findings from this study provides evidence from participants of this value and quantified what they gain from this experience.

Three authors have participated in an exchange during their GP training. They found the experience enriched their training, providing insight into primary health systems and general practice as a specialty that were not provided elsewhere in their training. All were inspired to help provide these experiences for others and evaluate the impact of exchange. One reviewer was unfamiliar with, and had not completed an exchange. All reviewers' independent data analysis was comparable regardless of experience.

This study focused on a small group of UK participants who undertook this experience within a set time frame. These early career GPs also volunteered their time to participate in these exchanges and would presumably have had some positive bias towards this experience prior 
to starting the exchange. We recognise therefore that these views may not represent the views of all early career GPs nor would it represent the views of international colleagues. Future research could indeed compare and contrast experiences from both exchange participants so that international views could be taken into account.

\section{Conclusion}

Reflective practice is a keystone within general practice teaching and training within the UK. Observation and comparison of general practice in the UK versus European countries provided insightful reflection and potential improvements and innovation within UK practice. (Word count 2434301)

\section{References}

1. Johnson J, Regets M. International Mobility of Scientists and Engineers to the United States - Brain Drain or Brain Circulation? 1998 SRS Issue Brief.

2. Dessoff A. Who’s NOT Going Abroad? Inter Educ 2006;15(2):20-27.

3. Corbett A. Book review : Internationalisation of Higher Education and Global mobility. ERIS - Eur Rev Inter Stud. 2014;1(3):165-168.

4. Volet E, Ang G. Culturally Mixed Groups on International Campuses: an Opportunity for Inter-cultural Learning. High Educ Res Dev. 2006;17(1):5-23.

5. Barata A, Rigon S. Family medicine $360^{\circ}$ : Global exchanges in family medicine. J Family Med Prim Care. 2015;4(3):305-30.

6. Editorial. The Globalisation of the NHS. Lancet 2002;350:1442-1448.

7. Abdullah S. Towards an individualised clients care: implications for education. The transcultural approach. J Adv Nur. 1995;22:715-20. 
8. Jones L. The Social Context of Health and Health work. Basingstoke : Macmillan Press Ltd; 1994.

9. www.eacea.ec.europa.eu accessed 29/10/17.

10. Charmaz K. Grounded theory: A practical guide through qualitative analysis. Thousand Oaks, California, Sage; 2006.

11. Frenk J, Chen L, Bhutta Z et al. Health professionals for a new century: transforming education to strengthen health systems in an interdependent world. Lancet. 2010;37:1923-58.

12. Lyratzopoulos G, Elliott M, Barbiere J, et al. Understanding ethnic and other sociodemographic differences in patient experience of primary care: evidence from the English General Practice Patient Survey. BMJ Qual Saf. 2012;21(1):21-29.

13. Deutsch K, Burrel S, Kann R, et al. Political Community and the North Atlantic Area. Princeton, NJ: Princeton University Press; 1968.

14.13. Sigalas E. Cross-border mobility and European identity: The effectiveness of intergroup contact during the ERASMUS year abroad. Eur Un Pol. 2010;11(2):241265.

15.14. Kitamura K, Fetters M, Ban N. The Experiences of Japanese Generalist Physicians in Overseas Faculty Development Program. Inter Fam Med. 2002;34(10):761-5.

16.15. Wearne E. A mirror or a window? The Irish registrar exchange program. (2008) Aus Fam Phy. 2008;37(3):158-9.

17.16. Jelley D. (2002) A UK-Portugal general practice exchange programme, Eur J Gen Prac. 2002;8(2):75-7.

18.17. Munir S, Hollister C. (2013) International out of programme experience (OOPE): is it worthwhile? Ed Prim Car. 2013;24(5):303-307. 
19.18. Yin RK. Qualitative research from start to finish. New York: Guilford Press;

2011.

20.19. https://gprecruitment.hee.nhs.uk/Recruitment/GHF accessed 18/3/17 


\section{Appendix 1 - Exert from the exchange participant document, outlining the requirements for the exchange report.}

We kindly ask that reports are interesting and self-reflective of your own learning experiences. Please avoid producing a report that is not self-reflective i.e. purely relays facts which can be found elsewhere online. The minimum word count is 500 words.

To help you, these are some topics you may choose to write about:

- A comparison between your own and your Host's(/Visitor's) country’s:

o Undergraduate training

o General Practice Training

o Role of the GP trainee

o Role of the GP

o Role of the other members of the clinic e.g. nurses, psychologist etc.

o Patient expectations

o Working hours

o Appointment length

o Number of patients seen in a day

o GP Clinic consultation rooms \& building structure

o Additional services offered o Home visits

o Acute care management

o Chronic care management

o GP pay

o GP insurance

o How the General Practice is funded

o Relation between primary and secondary care

o GP Networks e.g. junior, research, quality, rural, education \& training

o How the GP/General Practice is viewed by society

o Culture \& Religion

o Law \& Ethics 
- What you think your country does well and what it could do better?

- What you learned from your experience?

- How your experience has affected you and the way you may work as a GP in future?

- Whether you would recommend this experience to someone else? 


\section{Biography}

\section{Dr Bernadeta Bridgwood}

After completing a PhD in Inorganic Chemistry, Bernadeta moved into medicine. Bernadeta is an Academic Clinical Fellow in General Practice. Her research interests include secondary stroke prevention, education in medicine and international primary care. Bernadeta currently leads the UK Erasmus plus exchange programme for junior general practitioners.

\section{Dr Eugene Tang}

After completing undergraduate and foundation medical training, Eugene took up Academic Clinical Fellowship position in General Practice, followed by an awarded for a NIHR Doctoral Research Fellowship. His research interests include dementia and earlier diagnosis through risk prediction models in primary care to identify those at highest risk, promoting academic general practice and international primary care. Eugene was the former Research lead of the Junior International Committee (JIC) of the Royal College of General Practitioners (RCGP).

\section{Dr Hannah Willoughby}

Hannah is a salaried GP, Merthyr Tydfil, formally an academic trainee in Wales deanery. She is both a participant in exchange and a former member of the UK exchange team through the RCGP JIC.

\section{Dr Madeleine Attridge}

Madeleine is a GP in Wales 
Denmark, Italy, Lithuania, first to final year and first

Netherlands, Poland, Male - 3

Portugal and Slovenia. year after completing training.

Table 1 - Summary of participant characteristics and countries visited (all participants $\mathrm{n}=16$ ) 